\title{
on Fluids
}

Focus on Fluids journals.cambridge.org/focus

\section{From snapshots to modal expansions - bridging low residuals and pure frequencies}

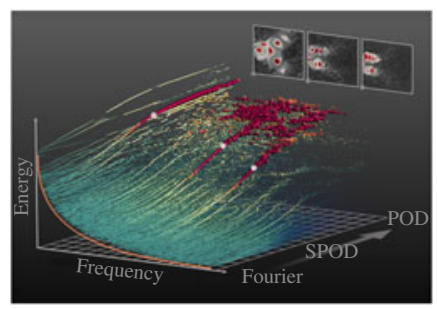

\author{
Bernd R. Noack ${ }^{1,2, \dagger}$ \\ ${ }^{1}$ LIMSI-CNRS, Rue John von Neumann, \\ Campus Universitaire d'Orsay, F-91403 Orsay, France \\ ${ }^{2}$ Institut für Strömungsmechanik, Technische Universität \\ Braunschweig, Hermann-Blenk-Straße 37, \\ D-38108 Braunschweig, Germany
}

\begin{abstract}
Data-driven low-order modelling has been enjoying rapid advances in fluid mechanics. Arguably, Sirovich (Q. Appl. Maths, vol. XLV, 1987, pp. 561-571) started these developments with snapshot proper orthogonal decomposition, a particularly simple method. The resulting reduced-order models provide valuable insights into flow physics, allow inexpensive explorations of dynamics and operating conditions, and enable model-based control design. A winning argument for proper orthogonal decomposition (POD) is the optimality property, i.e. the guarantee of the least residual for a given number of modes. The price is unpleasant frequency mixing in the modes which complicates their physical interpretation. In contrast, temporal Fourier modes and dynamic mode decomposition (DMD) provide pure frequency dynamics but lose the orthonormality and optimality property of POD. Sieber et al. (J. Fluid Mech., vol. 792, 2016, pp. 798-828) bridge the least residual and pure frequency behaviour with an ingenious interpolation, called spectral proper orthogonal decomposition (SPOD). This article puts the achievement of the TU Berlin authors in perspective, illustrating the potential of SPOD and the challenges ahead.
\end{abstract}

Key words: low-dimensional models, nonlinear dynamical systems

\section{Introduction}

Fluid mechanics can provide flow descriptions with a widely varying level of detail. In engineering applications, one may just need one quantity like a pressure loss in a pipe flow or lift of an airfoil, regardless of the flow physics. In contrast,

\section{$\dagger$ Email address for correspondence: Noack@limsi.fr}

The figure by the title illustrates the SPOD interpolation between POD and Fourier modes.

Courtesy of M. Sieber et al. (see http://dx.doi.org/10.1103/APS.DFD.2015.GFM.P0007). 
direct numerical simulations of turbulence are challenged by the need for a fine mesh resolving the Kolmogorov scale. The number of grid points scales with the Reynolds number $R e^{9 / 4}$. In contrast, many laminar and turbulent flows exhibit coherent structures living in a much lower dimensional state space. These coherent structures inspired Leonardo da Vinci's famous drawings of a wake and were later quantified by Eulerian and Lagrangian topology concepts. Low-dimensional coherent structure descriptions, or feature extraction in computer-science language, are a core discipline of theoretical fluid mechanics.

In the 19th century, unsteady flows were predominantly explored with Lagrangian vortex models based on Helmholtz laws. Most applications were 2-D flows, like vortex pairing, von Kármán vortex shedding, axisymmetric jets and shear layers. In 1915, B. G. Galërkin developed a Eulerian modelling path based on expansions with global modes (see e.g. Fletcher 1984). After initial applications to vibrations in solid mechanics, these Galerkin models enjoyed increasing popularity in fluid mechanics. Burkardt, Gunzburger \& Lee (2006) advocated cluster-based reduced-order modelling, a novel approach that has become increasingly popular nowadays. The discussion of reduced-order modelling approaches could easily be extended.

The most popular variants of Galerkin models are based on empirical expansions as 'post-mortem' processing of computational or experimental snapshot data. In the following, we assume a stationary observation domain $\Omega$, a space-time dependent velocity field $\boldsymbol{u}(\boldsymbol{x}, t)$, a solution for a single operating condition and a simple manipulation or filter $\boldsymbol{v}=H(\boldsymbol{u})$, e.g. the subtraction of a base flow $\boldsymbol{u}^{B}$. The observable $\boldsymbol{v}$ and domain $\Omega$ define an inner product $(\cdot, \cdot)_{\Omega}$ in the Hilbert space of square-integrable functions $L^{2}(\Omega)$ and the associated norm $\|\cdot\|_{\Omega}$. Virtually all expansions have the form

$$
\boldsymbol{v}(\boldsymbol{x}, t)=\sum_{i=1}^{N} a_{i}(t) \boldsymbol{u}_{i}(\boldsymbol{x})+\boldsymbol{\epsilon}(\boldsymbol{x}, t), \quad \text { where } \boldsymbol{u}_{i}(\boldsymbol{x})=\sum_{m=1}^{N} \boldsymbol{T}_{i}^{m} \boldsymbol{v}^{m}(\boldsymbol{x}) .
$$

Here, the flow is expanded with $N$ time-dependent amplitudes $a_{i}$ and space-dependent modes $\boldsymbol{u}_{i}$, which are linear combinations of the snapshots $\boldsymbol{v}^{m}$ at equidistantly sampled time $t^{m}$. $\boldsymbol{\epsilon}$ denotes the expansion residual. Furthermore, if $\boldsymbol{v}$ represents the flow state, there exists an autonomous dynamics

$$
\boldsymbol{a}^{m+1}=\boldsymbol{F}\left(\boldsymbol{a}^{m}\right) \quad \text { where } \boldsymbol{a}=\left(a_{1}, \ldots, a_{N}\right)^{t} .
$$

A key design parameter is the matrix $\boldsymbol{T}_{i}^{m}$ which gears down $M$ snapshots into a smaller number $N$ of the most relevant modes. This design parameter can serve several goals. A natural choice is to minimize the average residual $M^{-1} \sum_{m=1}^{M}\left\|\epsilon^{m}\right\|_{\Omega}^{2}$. This leads to orthogonal POD modes. POD tends to mix frequencies in each mode and thus complicate the physical interpretation. Second, pure frequency modes can be extracted by a Fourier transform $\boldsymbol{T}_{i}^{m} \sim \mathrm{e}^{i \omega_{i} t^{m}}, \omega_{i}$ being the frequency of the $i$ th mode. In this case, the $a_{i}$ have, by design, harmonic behaviour, but the spatial orthogonality of the modes is generally lost. Third, linear dynamics $\boldsymbol{F}(\boldsymbol{a})=\boldsymbol{A} \boldsymbol{a}$ may be assumed, and corresponding eigenmodes identified. The third goal leads to DMD (Rowley et al. 2009; Schmid 2010). With suitable transients some of the DMD modes are stability eigenmodes. For well-resolved post-transient data, DMD modes approximate Fourier modes. Moreover, the first $N$ DMD modes may be chosen to minimize the averaged expansion residual (Chen, Tu \& Rowley 2012). 


\section{Overview}

The paper by Sieber, Paschereit \& Oberleithner (2016) proposes a novel interpolation between POD and Fourier modes. Their first ingenious step is to discover the POD eigenvalue problem with the snapshot correlation matrix $\boldsymbol{C}^{m n}=\left(\boldsymbol{v}^{m}, \boldsymbol{v}^{n}\right)_{\Omega}$ as a design parameter. Secondly, they employ a relation between the autocorrelation function and the correlation matrix $\boldsymbol{C}^{m n}$. This relation gave rise to the idea of the filter that leads to periodic mode amplitudes in the limit of maximum filtering. A third important step was to derive the effect on the dynamics $\boldsymbol{F}$ from a filtered correlation matrix. The resulting filtered eigenvalue problem can continually interpolate between POD modes (no filtering) and Fourier modes (maximal filtering). In other words, minimal residual and orthonormality can be traded against harmonic behaviour of the mode amplitudes. This modal interpolation is impressively demonstrated by particle image velocimetry (PIV) data from three experiments: a swirling jet undergoing vortex breakdown, an airfoil with Gurney flap and a fluidic oscillator.

The key idea of interpolating modes by interpolating the eigenvalue problem is elegant but not new. In bifurcation analysis, the change of the spectral characteristics of the stability matrix with respect to the order parameter is a standard procedure. For data-driven approaches, an interpolated eigenvalue problem has been employed to interpolate between POD and stability eigenmodes (Morzyński et al. 2006). The second key enabler, which makes the temporal correlation matrix translation invariant with respect to time, has a spatial analogue. George (1999) considered a space-translation invariant cross-correlation and arrived at spatial Fourier modes for the corresponding POD problem. The third enabler, which incorporates the dynamics $\boldsymbol{F}$, is key to the construction of DMD modes. The achievement of the authors is bringing all enablers together to explore an important new design opportunity in modal expansions.

\section{Future}

The literature on the proposed empirical modal expansion is much richer than for the POD and Fourier modes. The modal expansion may be geared towards an objective function (Hoarau et al. 2006). Another direction may be sparse dynamics (Brunton, Proctor \& Kutz 2016). To some extent DMD for post-transient data yields a sparse dynamics with a system of oscillators. One may also add Navier-Stokes-based balance equations as a constraint (Balajewicz, Dowell \& Noack 2013). The list of modal expansions is as rich as the goals for which reduced-order models are used. For the interpolation between expansions for different goals, the underlying eigenproblems constitute, again, an elegant starting point.

There are still big challenges for modal expansions and associated Galerkin models - all arising from the fact that fluid flows do not behave like the membrane that B. G. Galërkin considered. First, low-order flow descriptions may require 'flexible' modes. A cylinder wake transient from the steady solution to periodic vortex shedding shows a slow metamorphosis from far-wake fluctuations characterized by stability modes to near-wake fluctuations accurately presented by POD modes. Elegant extractions of such flexible modes are pursued by Iollo \& Lombardi (2014) and Babaee \& Sapsis (2016). Second, low-order expansions may require addition or removal of modes depending on the flow state and operating condition. Clustering may serve these purposes (Kaiser et al. 2014). Yet, an elegant general-purpose framework is still missing. Third, a key challenge of modal expansions and associated Galerkin models is rarely reflected in the literature. The very idea of modal 
expansions implies a coherency in space-time behaviour. Equation (1.1) is based on an 'elliptical' coherency which may be characterized by the cross-correlation function $\boldsymbol{R}(\boldsymbol{x}, \boldsymbol{y})=\overline{\boldsymbol{u}(\boldsymbol{x}, t) \otimes \boldsymbol{u}(\boldsymbol{y}, t)}$. If one knows the flow $\boldsymbol{u}$ in a tiny neighbourhood of $\boldsymbol{x}$, one can determine all mode amplitudes from (1.1) and thus the flow behaviour everywhere. This ansatz might be justified for post-transient closed flows or absolutely unstable flow regions. For shear flows, a far more realistic prediction can be made by considering the convection process. This 'hyperbolic' coherency is reflected in Taylor's frozen flow hypothesis and can be quantified in the space-time cross-correlation function $\boldsymbol{R}(\boldsymbol{x}, \boldsymbol{y}, \tau)=\overline{\boldsymbol{u}(\boldsymbol{x}, t) \otimes \boldsymbol{u}(\boldsymbol{y}, t-\tau)}$. The convection effect could be incorporated in a Floquet-like expansion $\boldsymbol{v}(\boldsymbol{x}, t)=\sum_{i=1}^{N} a_{i}(t) \boldsymbol{u}_{i}(\boldsymbol{x}, t)$ with time-dependent modes representing convecting flow structures and slowly varying $a_{i}$. In principle, the POD procedure for the space-time cross-correlation function can distill these modes.

Looking at recent progress, one can expect dramatic changes in data-driven low-dimensional modelling to incorporate the dynamics, mode deformations, mode 'scheduling/switching' and convective effects, largely fuelled by the powerful methods of machine learning. It is also natural to expect that we will see first many uncorrelated advances for subproblems and problem-related 'cook book' recipes before unifying optimization principles for well-defined classes of problems emerge. Last, but not least, the goal of reduced-order modelling should always be kept in mind, but this may be the topic of a future Focus on Fluids article.

\section{References}

BABAeE, H. \& SAPsis, T. P. 2016 A variational principle for the description of time-dependent modes associated with transient instabilities. Phil. Trans. R. Soc. Lond. 472 (2186), 20150779.

Balajewicz, M., Dowell, E. H. \& NoACK, B. R. 2013 Low-dimensional modelling of high-Reynolds-number shear flows incorporating constraints from the Navier-Stokes equation. J. Fluid Mech. 729, 285-308.

Brunton, S. L., Proctor, J. L. \& Kutz, N. J. 2016 Discovering governing equations from data by sparse identification of nonlinear dynamical systems. Proc. Natl Acad. Sci. USA 113 (5), 3932-3937.

Burkardt, J., Gunzburger, M. D. \& Lee, H.-C. 2006 Centroidal Voronoi tessellation-based reduced-order modeling of complex systems. SIAM J. Sci. Comput. 28 (2), 459-484.

Chen, K. K., TU, J. H. \& Rowley, C. W. 2012 Variants of dynamic mode decomposition: boundary condition, Koopman, and Fourier analyses. J. Nonlinear Sci. 22 (6), 887-915.

Fletcher, C. A. J. 1984 Computational Galerkin Methods, 1st edn. Springer.

George, W. K. 1999 Some thoughts on similarity, the POD, and finite boundaries. In Fundamental Problematic Issues in Turbulence (ed. A. Gyr, W. Kinzelbach \& A. Tsinober), Birkhäuser.

Hoarau, C., Borée, J., Laumonier, J. \& Gervais, Y. 2006 Analysis of the wall pressure trace downstream of a separated region using extended proper orthogonal decomposition. Phys. Fluids 18, 055107.

Iollo, A. \& Lombardi, D. 2014 Advection modes by optimal mass transfer. Phys. Rev. E 89, $002923,1-12$.

Kaiser, E., Noack, B. R., Cordier, L., Spohn, A., Segond, M., Abel, M. W., Daviller, G., Östh, J., Krajnović, S. \& Niven, R. K. 2014 Cluster-based reduced-order modelling of a mixing layer. J. Fluid Mech. 754, 365-414.

Morzyński, M., Stankiewicz, W., Noack, B. R., Thiele, F. \& TAdmor, G. 2006 Generalized mean-field model for flow control using continuous mode interpolation. AIAA Paper 2006-3488.

Rowley, C. W., Mezić, I., Bagheri, S., Schlatter, P. \& Henningson, D. S. 2009 Spectral analysis of nonlinear flows. J. Fluid Mech. 645, 115-127.

SCHMID, P. J. 2010 Dynamic mode decomposition for numerical and experimental data. J. Fluid. Mech. 656, 5-28.

Sieber, M., Paschereit, C. O. \& Oberleithner, K. 2016 Spectral proper orthogonal decomposition. J. Fluid Mech. 792, 798-828. 